\title{
Insulin Resistance Index and Proatherogenic Lipid Indices in the Offspring of People with Diabetes
}

\author{
Oyebola Oluwagbemiga Sonuga ${ }^{a}$ Fayeofori Mpakaboari Abbiyesuku ${ }^{a}$ \\ Kayode Samson Adedapo ${ }^{a}$ Ayobola Abimbola Sonuga ${ }^{b}$

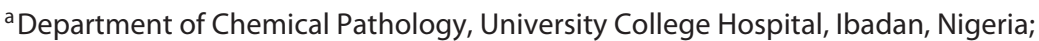 \\ ${ }^{b}$ Department of Science Laboratory Technology, Ekiti State University, Ado Ekiti, Nigeria
}

\section{Keywords}

Diabetes mellitus · Atherogenic lipid indices · Insulin resistance $\cdot$ Cardiovascular disease

\begin{abstract}
Background: Diabetes mellitus and cardiovascular diseases significantly contribute to medical morbidity and mortality worldwide, especially in developing countries like Nigeria. Insulin resistance, a characteristic finding of type 2 diabetics and their offspring, is associated with an abnormal lipid metabolism and cardiovascular disease. Aims: This study therefore aims to determine the pattern of lipid biomarkers of atherogenesis and their relationship with insulin resistance indexin young people with a family history of diabetes mellitus. Methods: This is a cross-sectional study carried out among 150 apparently healthy young adults between 18 and 25 years of age, including 76 with a family history of diabetes mellitus in first- and/or second-degree relatives (YWFH) and 74 with no family history of diabetes mellitus (YWoFH). Anthropometric characteristics, insulin resistance index, plasma glucose, fasting lipid profile (plasma total cholesterol, triglycerides [TG], high-density lipoprotein cholesterol, low-
\end{abstract}

density lipoprotein cholesterol), and serum levels of insulin, lipoprotein(a) [Lp(a)], apolipoprotein B (ApoB), apolipoprotein A-1 (ApoA-1), and ApoB/ApoA-1 ratios were compared in the 2 groups. Plasma glucose, total cholesterol, high-density cholesterol, and TG were measured using standard methods. The Friedewald equation was used to calculate low-density cholesterol. Serum insulin, $L p(a)$ levels, ApoB, and ApoA-1 were also measured using standard assays. The insulin resistance index was determined using homeostatic model assessment (HOMA). Statistical analysis was performed using SPSS version 20.0. Comparisons between variables were performed using the Mann-Whitney $U$ test, and correlations between variables were performed using Spearman rank correlation coefficients. The level of significance was set at $p<0.05$. Results: Offspring of diabetics (YWFH) had a significantly higher median BMI $(p=0.015)$, waist-tohip ratio (WHR; $p=0.002)$, insulin resistance index $(p=0.038)$, total cholesterol $(p=0.017), \mathrm{TG}(p=0.004), \operatorname{Lp}(\mathrm{a})(p=0.045)$, $\operatorname{ApoB}(p=0.002)$, and ApoB/ApoA-1 ratio $(p=0.001)$ than the age-matched control group with no family history of diabetes mellitus (YWoFH). There was no correlation between the insulin resistance index and each of the lipid biomarkers of atherogenesis except $L p(a)$, with which it was negatively cor-

\begin{tabular}{|c|c|}
\hline KARGER & $\begin{array}{l}\text { (c) } 2019 \text { The Author(s) } \\
\text { Published by S. Karger AG, Basel }\end{array}$ \\
\hline $\begin{array}{l}\text { E-Mail karger@karger.com } \\
\text { www.karger.com/ijd }\end{array}$ & $\begin{array}{l}\text { This article is licensed under the Creative Commons Attribution- } \\
\text { NonCommercial-NoDerivatives } 4.0 \text { International License (CC BY- } \\
\text { NC-ND) (http://www.karger.com/Services/OpenAccessLicense). } \\
\text { Usage and distribution for commercial purposes as well as any dis- } \\
\text { tribution of modified material requires written permission. }\end{array}$ \\
\hline
\end{tabular}

Oyebola Oluwagbemiga Sonuga

Department of Chemical Pathology, University College Hospital Queen Elizabeth Road, PMB 5116

Ibadan, Oyo State (Nigeria)

E-Mail oyebolasonuga@yahoo.com 
related. Conclusion: This study demonstrates that a positive family history of type 2 diabetes mellitus is associated with a higher insulin resistance index and elevated atherogenic lipid indices; thus, a positive family history of diabetes mellitus in first or second-degree relatives when the index person is not diabetic confirms a significant cardiovascular risk.

(c) 2019 The Author(s)

Published by S. Karger AG, Basel

\section{Introduction}

Diabetes mellitus significantly contributes to medical morbidity and mortality worldwide, especially in developing countries like Nigeria [1]. Non-communicable diseases, of which cardiovascular diseases are the most prevalent, cause the greatest morbidity and mortality worldwide. Resistance to insulin-stimulated glucose uptake is a characteristic finding in patients with type 2 diabetes mellitus and it has also been reported among offspring and relatives of diabetics. Insulin resistance is a predisposing factor for hypertension, dyslipidaemia, hypercoagulability, endothelial dysfunction, albuminuria, and premature cardiovascular disease.

Although the end points for cardiovascular risk are not usually seen in childhood, the components of the metabolic syndrome (obesity, hypertension, dyslipidaemia, and hyperinsulinaemia) track from childhood into adulthood, which supports the conclusion that the precursors of cardiovascular disease are present early in life. Therefore, it is necessary to establish a robust and precise risk indicator for lipid imbalance and atherogenesis in childhood and young adulthood, in order to prevent or delay the progression of diabetes mellitus and its complications, especially cardiovascular disease.

However, despite more aggressive interventions for lowering low-density lipoprotein cholesterol (LDL-C) levels, the majority of coronary artery disease events still occur, perhaps related to the fact that intervention was not started earlier in life or that LDL-C levels represent an incomplete picture of atherogenic potential [2]. Measurement of apolipoprotein B (ApoB) has been shown to outperform LDL-C and non-high-density lipoprotein cholesterol (HDL-C) as a predictor of coronary artery disease events and as an index of residual coronary artery disease risk $[3,4]$. It is therefore recommended that $A$ poB and the ApoB/apolipoprotein A-1 (ApoA-1) ratio are determined after the measurement of LDL-C, non-HDL-C, and the ratio of total cholesterol/HDL-C to better predict coronary artery disease and assess the efficacy of treatment [5]. Also, the association between lipoprotein(a) [Lp(a)] and coronary heart disease is well established [6], but the potential relationship of these lipid indices to the insulin resistance syndrome remains to be clarified among offspring of diabetics, with only little available published data.

We hypothesise that there is an association between the insulin resistance index (a measure of insulin sensitivity) and lipid markers of atherogenesis in the offspring of diabetics, and that the level of these lipid markers will be significantly higher compared with their age-matched controls (offspring with no diabetic history).

\section{Materials and Methods}

This is a cross-sectional study carried out among age-matched students of a higher institution in Ibadan over a period of 8 months. A convenient (non-random) sampling technique was used to select the study participants. A total of 150 apparently healthy participants with an age range from 18 to 25 years were recruited for this study; 76 subjects had a family history of diabetes mellitus among their first- and/or second-degree relatives (YWFH), and 74 were controls, without a family history of diabetes mellitus (YWoFH). Ethical approval was obtained from the University of Ibadan/University College Hospital Ibadan Health Research Ethics Committee (No. UI/EC/16/0301).

\section{Inclusion Criteria}

(i) Apparently healthy individuals between the age of 18 and 25 years, with or without a family history of diabetes mellitus among their first- and/or second-degree relatives.

(ii) Voluntary consent to participate in the study.

\section{Exclusion Criteria}

(i) Overweight individuals (BMI >25.0) or with obesity, diabetes mellitus, or any known endocrinopathies because of abnormal glucose/insulin homeostasis and insulin resistance in them.

(ii) Smokers, because glucose concentration is higher in smokers than non-smokers and glucose tolerance is mildly impaired in smokers. There is also delayed insulin release in response to glucose in smokers.

(iii) Pregnant or lactating women and subjects with chronic illness or on chronic steroid/lipid-lowering medication were excluded because of their influence on the carbohydrate metabolism.

(iv) Individuals with hypertension, on antihypertensive medication, or with blood pressure $\geq 140 / 90 \mathrm{~mm} \mathrm{Hg}$.

(v) History of cardiovascular diseases (coronary artery disease, congestive heart failure, stroke or transient ischemic attack, and intermittent claudication).

(vi) Refusal of consent.

\section{Data Collection}

A data collection form (questionnaire) which contained items on the demographic characteristics, clinical measurements, medical and family history of diabetes mellitus, diet history, and results of the laboratory analysis of participants was used for study recruitment. Data collection was by personal interview and an in- 
formed written consent form was obtained from all participants after educating them on the benefits and relevance of the study.

The clinical measurements included weight (in kilograms to 1 decimal place) and height (in metres to 2 decimal places) were obtained with the participants wearing light clothing without shoes, by stadiometer and the stadiometer's head piece, respectively. BMI was calculated as weight $/$ height $^{2}\left(\mathrm{~kg} / \mathrm{m}^{2}\right.$ to 1 decimal place). Waist circumference was measured in centimetres to 1 decimal place at the midpoint between the lower margin of the least palpable rib and the top of the iliac crest (umbilicus) with the tape around the body in a horizontal position, with the measurer standing at the side of each participant. Hip circumference (in centimetres to 1 decimal place) was measured at the widest portion of the buttocks or greatest hip girth (greater trochanter). Body fat distribution was assessed indirectly by the waist-to-hip ratio (WHR to 2 decimal places). Blood pressure was measured twice, using a standard aneroid sphygmomanometer on the left arm after at least $10 \mathrm{~min}$ of rest with the average of the measurements recorded.

\section{Specimen Collection and Storage}

The procedure was carried out at the Metabolic Research Ward, University College Hospital Ibadan. An oral glucose tolerance test (OGTT) was performed in the morning between 7 a.m. and 9 a.m., after 3 days of usual daily diet and activity, and after 10-12 h of overnight fast by each participant. Twelve millilitres of blood were drawn from each participant in the fasting state from the antecubital vein, with $4 \mathrm{~mL}$ dispensed into an EDTA bottle for the fasting lipid profile, $4 \mathrm{~mL}$ into a gel clot/activator bottle for other lipid studies and fasting insulin measurement, and $4 \mathrm{~mL}$ into a fluoride oxalate bottle for fasting glucose measurement. Thereafter, $410 \mathrm{~mL}$ of a 75-g glucose solution was administered to each participant orally over a 5-min period. Eight millilitres of venous blood was obtained from each participant, for 2 -h post-OGTT glucose and 2 -h post-OGTT insulin measurement ( $4 \mathrm{~mL}$ each). Specimen bottles were transported immediately after blood collection in ice packs to the laboratory. Each specimen bottle was centrifuged at 3,000 $\mathrm{g}$ for $15 \mathrm{~min}$ within $30 \mathrm{~min}$ of blood collection. Full clot retraction of the blood sample in the gel clot/activator bottle was allowed before centrifuging. Both the serum and plasma were decanted into their respective labelled plain bottles and stored at $-20^{\circ} \mathrm{C}$ for not more than 3 months before being analysed.

\section{Assay Methods}

Plasma glucose was measured using the glucose-oxidase method on an automated chemistry analyser LW C 100 plus. Enzymatic methods were also used to analyse plasma total cholesterol (cholesterol-oxidase), HDL-C (direct HDL PEGME), and triglycerides (TG; lipase/glycerol phosphate-oxidase) on an automated chemistry analyser (LW C 100 plus). The Friedewald equation was used to calculate LDL-C. Serum insulin and Lp(a) levels were manually measured using the ELISA method, while serum ApoB and ApoA-1 were measured using immunoturbidimetric assays on an automated chemistry analyser (LW C 100 plus). The insulin resistance index was calculated using the University of Oxford HOMA calculator software, version 2.2. HOMA-IR was calculated as: [fasting glucose $(\mathrm{mmol} / \mathrm{L}) \times$ fasting insulin $(\mu \mathrm{IU} / \mathrm{mL})] \div 22.5$.

\section{Statistical Analysis}

The statistical analysis was performed using SPSS (Statistical Package for Social Sciences) version 20.0. The Kolmogorov-
Table 1. Anthropometric and clinical data of the YWFH and YWoFH groups

\begin{tabular}{lccl}
\hline & YWFH $(n=76)$ & YWoFH $(n=74)$ & $p$ \\
\hline Age, years & $20.0(19.0-24.0)$ & $21.0(20.0-23.0)$ & 0.137 \\
Height, m & $1.65(1.60-1.68)$ & $1.66(1.59-1.72)$ & 0.265 \\
Weight, kg & $60.0(53.0-67.4)$ & $58.5(52.8-62.0)$ & 0.166 \\
BMI & $22.4(19.7-24.6)$ & $20.5(19.3-23.0)$ & $0.015^{*}$ \\
WC, cm & $76.0(71.0-80.8)$ & $73.5(70.0-80.0)$ & 0.201 \\
HC, cm & $95.0(88.5-98.0)$ & $94.0(90.0-98.6)$ & 0.995 \\
WHR & $0.83(0.79-0.85)$ & $0.80(0.77-0.82)$ & $0.002^{*}$ \\
SBP, mm Hg & $120.0(100.0-120.0)$ & $110.0(100.0-120.0)$ & 0.154 \\
DBP, mm Hg & $80.0(66.3-80.0)$ & $70.0(60.0-80.0)$ & 0.050 \\
\hline
\end{tabular}

Values are presented as the median (IQR). ${ }^{*} p<0.05$. WC, waist circumference; HC, hip circumference; WHR, waist-to-hip ratio; SBP, systolic blood pressure; DBP, diastolic blood pressure.

Table 2. Fasting, 2-h post-OGTT glucose and insulin values of the YWFH and YWoFH groups

\begin{tabular}{lccc}
\hline & $\begin{array}{l}\text { YWFH } \\
(n=76)\end{array}$ & $\begin{array}{l}\text { YWoFH } \\
(n=74)\end{array}$ & $p$ \\
\hline FPG, mg/dL & $72.0(63.0-77.0)$ & $68.0(62.0-75.0)$ & 0.052 \\
2-h G, $\mathrm{mg} / \mathrm{dL}$ & $91.0(77.5-99.8)$ & $83.0(75.5-99.5)$ & 0.391 \\
Insulin F, $\mu \mathrm{IU} / \mathrm{mL}$ & $3.5(3.2-4.4)$ & $3.4(3.2-4.0)$ & 0.151 \\
Insulin 2 h, $\mu \mathrm{IU} / \mathrm{mL}$ & $33.0(17.3-42.8)$ & $23.6(16.6-41.0)$ & 0.230 \\
\hline
\end{tabular}

Values are presented as the median (IQR). FPG, fasting plasma glucose; 2-h G, 2-h post-OGTT plasma glucose; Insulin F, fasting serum insulin; Insulin $2 \mathrm{~h}, 2$-h post-OGTT serum insulin.

Smirnov test of normality revealed a non-Gaussian distribution (skewness: -3.054 to 4.256 ); hence, results are expressed as the median (interquartile range). Comparisons between median values of variables were performed using the Mann-Whitney U test for unpaired data. Correlations between the insulin resistance index and total cholesterol, HDL-C, TG, LDL-C, Lp(a), ApoB, ApoA-1, and ApoB/ApoA-1 were performed using Spearman rank correlation coefficients. The level of significance was taken to be $p<0.05$.

\section{Results}

\section{Demographic Characteristics of the Study Population}

A total of 150 apparently healthy students, with ages ranging from 18 to 25 years, were recruited for this study over a period of 8 months. Of the students, 76 belonged to the YWFH group, while the other 74 represented the YWoFH controls. 
Table 3. Comparison of insulin resistance index and lipid parameters between the YWFH and YWoFH groups

\begin{tabular}{lccc}
\hline & YWFH $(n=76)$ & YWoFH $(n=74)$ & $p$ \\
\hline HOMA-IR & $0.43(0.39-0.53)$ & $0.40(0.38-0.50)$ & $0.038^{*}$ \\
TC, mg/dL & $155.0(147.0-172.8)$ & $146.0(136.0-168.5)$ & $0.017^{*}$ \\
HDL-C, mg/dL & $60.0(53.0-68.0)$ & $59.0(51.0-66.3)$ & 0.353 \\
TG, mg/dL & $71.0(59.3-90.3)$ & $62.5(54.0-76.0)$ & $0.004^{*}$ \\
LDL-C, mg/dL & $83.0(71.0-99.3)$ & $74.5(65.8-96.3)$ & 0.128 \\
Lp(a), mg/dL & $80.0(61.0-89.0)$ & $71.0(55.5-83.0)$ & $0.045^{*}$ \\
Apo B, mg/dL & $87.0(81.0-99.0)$ & $78.5(69.0-96.3)$ & $0.002^{*}$ \\
Apo A-1, mg/dL & $167.0(157.0-177.0)$ & $168.0(160.5-181.0)$ & 0.147 \\
ApoB/ApoA-1 & $0.52(0.46-0.59)$ & $0.48(0.40-0.53)$ & $0.001^{*}$ \\
\hline
\end{tabular}

Values are presented as the median (IQR). ${ }^{*} p<0.05$. HOMA-IR, insulin resistance index; TC, total cholesterol; HDL-C, high-density lipoprotein cholesterol; TG, triglycerides; LDL-C, low-density lipoprotein cholesterol; Lp(a), lipoprotein(a); ApoB, apolipoprotein B; ApoA-1, apolipoprotein A-1.

Table 4. Correlation between the insulin resistance index (HOMAIR), clinical data, and lipid parameters for YWFH subjects

\begin{tabular}{lrl}
\hline & \multicolumn{2}{l}{ YWFH } \\
\cline { 2 - 3 } & $r$ & $p$ \\
\hline HOMA-IR vs. BMI & 0.007 & 0.952 \\
HOMA-IR vs. WHR & 0.105 & 0.366 \\
HOMA-IR vs. SBP & 0.274 & $0.017^{*}$ \\
HOMA-IR vs. DBP & 0.075 & 0.522 \\
HOMA-IR vs. total cholesterol & -0.020 & 0.861 \\
HOMA-IR vs. HDL-C & 0.066 & 0.570 \\
HOMA-IR vs. TG & 0.200 & 0.083 \\
HOMA-IR vs. LDL-C & -0.049 & 0.673 \\
HOMA-IR vs. Lp(a) & -0.456 & $0.000^{*}$ \\
HOMA-IR vs. ApoB & 0.003 & 0.980 \\
HOMA-IR vs. ApoA-1 & -0.032 & 0.783 \\
HOMA-IR vs. ApoB/ApoA-1 ratio & 0.015 & 0.899 \\
\hline
\end{tabular}

$* p<0.05$. WHR, waist-to-hip ratio; SBP, systolic blood pressure; DBP, diastolic blood pressure; FPG, fasting plasma glucose; FSI, fasting serum insulin; Lp(a), lipoprotein(a); ApoB, apolipoprotein B; ApoA-1, apolipoprotein A-1.

Forty-four (59.5\%) of the YWoFH group were females while $30(40.5 \%)$ were males; of the YWFH group, 43 $(56.6 \%)$ were females and $33(43.4 \%)$ were males. The female-to-male ratios were similar in both groups (1.3:1) and there was no significant difference in the numbers of females and males in the 2 groups. The median age of the YWFH group (20.0 years, range 19.0-24.0) was not significantly different from the YWoFH group (21.0 years, range $20.0-23.0 ; p=0.137$ ).

\section{Anthropometric and Clinical Data of YWFH and YWoFH}

The BMI and WHR of the YWFH group were significantly higher than those of the YWoFH group ( $p=0.015$ and 0.002 , respectively). There was no significant difference in the median weight, height, waist circumference, hip circumference, systolic blood pressure, and diastolic blood pressure between the 2 groups (Table 1 ).

\section{Fasting, 2-H Post-OGTT Glucose and Insulin Values of $Y W F H$ and $Y W o F H$}

There were no significant differences between the median fasting plasma glucose $(p=0.052), 2$-h post-OGTT plasma glucose $(p=0.391)$, fasting serum insulin $(p=$ $0.151)$, and 2 -h post-OGTT serum insulin $(p=0.230)$ of the YWFH and YWoFH groups (Table 2).

\section{Comparison of Insulin Resistance Index and Lipid \\ Parameters between YWFH and YWoFH}

The YWFH subjects had a significantly higher insulin resistance index than the YWoFH controls $(p=0.038)$. The median total cholesterol, TG, Lp(a), ApoB, and ApoB/ApoA-1 ratio of the YWFH group were significantly higher than those of the YWoFH group $(p=0.017$, $p=0.004, p=0.045, p=0.002$, and $p=0.001$, respectively). There was no significant difference in the median HDLC, LDL-C, and ApoA- 1 between the 2 groups (Table 3 ).

\section{Correlation between Insulin Resistance Index, Clinical} Data, and Lipid Parameters of YWFH

There was a significant positive correlation between the insulin resistance index and systolic blood pressure 


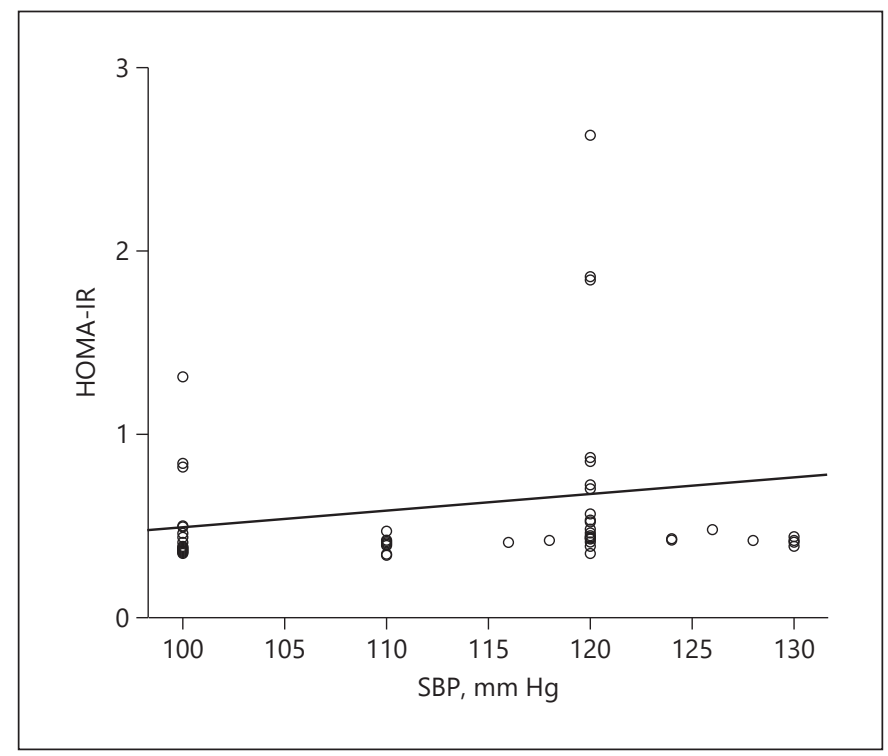

Fig. 1. Correlation between insulin resistance index (HOMA-IR) and systolic blood pressure (SBP) in the YWFH group. $r=0.274$, $p=0.017 ; R^{2}=0.031$, adjusted $R^{2}=0.018, p=0.127$.

$(r=0.274, p=0.017)$, which became insignificant after using $R^{2}$ and adjusted $R^{2}\left(R^{2}=0.031\right.$; adjusted $R^{2}=0.018$, $p=0.127)$. There was also a significant negative correlation between the insulin resistance index and $\mathrm{Lp}(\mathrm{a})$ of the YWFH group $(r=-0.456, p=0.00)$. There was no correlation between the insulin resistance index and other measured variables. This is represented in Table 4. Figure 1 shows the correlation between the insulin resistance index and systolic blood pressure in the YWFH group, and Figure 2 shows the correlation between the insulin resistance index and Lp(a) in the YWFH group.

\section{Discussion}

Several reports [7-9] have shown that insulin resistance (with resultant hyperinsulinaemia) is associated with dyslipidaemia (elevated TG and low HDL-C), atherosclerosis, type 2 diabetes, and premature cardiovascular diseases. Other studies [10-12] have also shown that offspring and relatives of type 2 diabetics have hyperinsulinaemia, despite being glucose tolerant. To this end, offspring of diabetics are more prone to cardiovascular diseases than offspring of non-diabetics. The present study looked at the level of lipid markers of atherogenesis in age-matched YWFH and YWoFH subjects, and the relationship of these markers with the insulin resistance in-

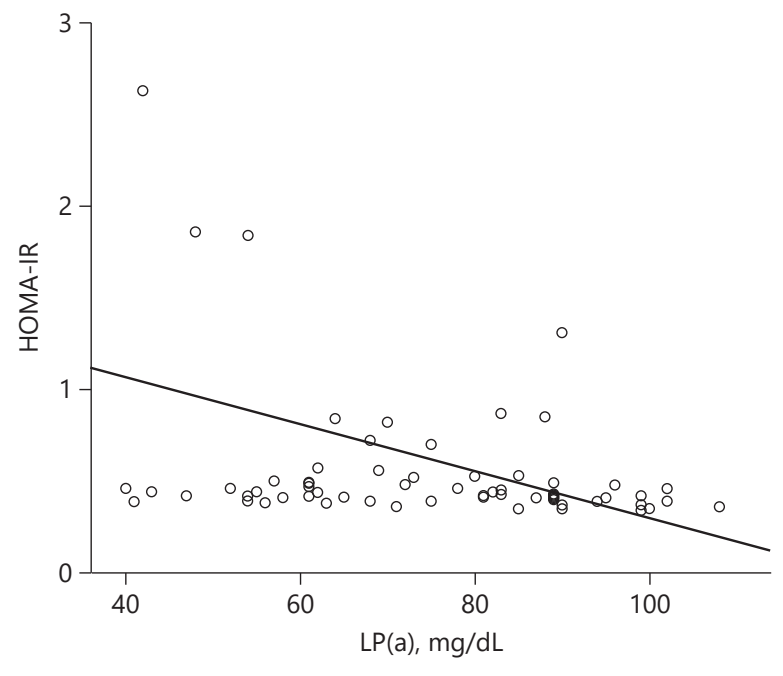

Fig. 2. Scatterplot representing the correlation between the insulin resistance index (HOMA-IR) and Lp(a) in the YWFH group. $r=$ $-0.456, p=0.000 ; R^{2}=0.211$.

dex in YWFH. It is important to stress that this study looked at an apparently healthy population yet to develop cardiovascular disease, diabetes, or metabolic syndrome. This fact explains why their biochemical results were within clinical decision limits, yet with clear and significant differences between the YWFH and YWoFH groups.

The study concluded that YWFH subjects have a significantly higher BMI than the age-matched $\mathrm{YWoFH}$ group. This is similar to the findings of Adeleye and Abbiyesuku [13] and Zafar et al. [14]. BMI is a measure of generalized obesity, and research has shown that there is an association between adiposity and insulin resistance in adults and children $[15,16]$. Therefore, from the present study, YWFH have more generalized body fat than YWoFH and are possibly at higher risk of resistance to insulin-stimulated glucose uptake.

It was observed in this study that the WHR of the YWFH subjects was significantly higher than in the YWoFH group. This observation is in agreement with the findings of Adeleye and Abbiyesuku [13] and Zafar et al. [14]. Other studies $[17,18]$ have shown that an increased WHR (a measure of abdominal or central obesity) is associated with the incidence of cardiovascular events and that it is a better predictor of cardiovascular risk than BMI. Thus, this present study shows that YWFH are more centrally obese than YWoFH, and are therefore likely to be at higher risk of developing resistance to insulin action 
and of experiencing cardiovascular events than YWoFH later in life.

In this study, YWFH had a significantly higher insulin resistance index (HOMA-IR) than YWoFH, which is in agreement with the findings of Chung et al. [19] and Shetty et al. [20]. This finding suggests that YWFH are at higher risk of biochemical and clinical disorders associated with insulin resistance.

The total cholesterol and TG of YWFH were significantly higher than those of YWoFH. Rahim et al. [21] and Moon et al. [22] reported similar findings, and concluded that lipid profiles of offspring were related to the diabetic parent's history. YWFH subjects had similar HDL-C values to the $\mathrm{YWoFH}$ controls, which is contrary to reports by Psyrogiannis et al. [23] and Rahim et al. [21]. However, in comparison to the present study, these studies investigated middle-aged populations, showing that decline in the HDL-C level is most likely an evolving phenomenon occurring over time in offspring of diabetics as they grow older. Hyperinsulinaemia is known to enhance hepatic very-low-density lipoprotein synthesis, which directly contributes to the increased plasma TG and LDL-C levels. Resistance to the action of insulin on lipoprotein lipase in peripheral tissues also contributes to elevated TG and LDL-C levels. Thus, compared to YWoFH, YWFH have higher levels of these atherogenic lipid particles, which may be as a result of the defective insulin action and higher serum insulin levels. Therefore, YWFH are at higher risk of cardiovascular disease even at this age.

The median values of $\mathrm{Lp}(\mathrm{a})$ in both groups were about 2- to 3-fold higher than the upper limit of reference interval for $\mathrm{Lp}(\mathrm{a})$, which is consistent with the report by Virani et al. [24] and Khera et al. [25]. They agreed that people of African descent have higher $\mathrm{Lp}(\mathrm{a})$ values compared to Caucasians, but do not manifest a higher incidence of coronary heart disease, because of the low prevalence of small ApoA isoforms in them. $\mathrm{Lp}(\mathrm{a})$ is an independent risk factor for coronary heart disease. The finding of significantly higher $\mathrm{Lp}(\mathrm{a})$ in YWFH is similar to the finding of Psyrogiannis et al. [23], indicating that YWFH are at higher risk of cardiovascular disease compared to YWoFH.

YWFH had a significantly higher ApoB concentration than $\mathrm{YWoFH}$, which is in concordance with the study by Hashemi et al. [26]. ApoB is a better predictor of cardiovascular risk than LDL-C and other non-HDL-C measures. Its higher level in YWFH compared to YWoFH in this study suggests that YWFH are at higher risk of cardiovascular disease.
The ApoB/ApoA-1 ratio combines these risk factors and examines them holistically with regard to cardiovascular risk. YWFH had a significantly higher ApoB/ApoA1 ratio than YWoFH. This observation is related to that of Savas Erdeve et al. [27], who reported that the ApoB/ ApoA-1 ratio was significantly higher in obese children with metabolic syndrome when compared to obese children without metabolic syndrome. An accumulating body of data indicates that the ApoB/ApoA-1 ratio is a powerful marker of risk for future cardiovascular disease $[4,28]$. The higher the value of the ApoB/ApoA-1 ratio, the more cholesterol is circulating in the plasma, and this cholesterol is likely to be deposited in the arterial wall, provoking atherogenesis and the risk of cardiovascular events. The lower the ApoB/ApoA-1 ratio, the lower is the plasma cholesterol reaching the periphery and the greater is the reverse cholesterol transport and other beneficial functions, and the lower is the risk of cardiovascular events. Thus, YWFH are at higher risk of cardiovascular events compared to YWoFH.

There was a modest significant positive correlation between the insulin resistance index and systolic blood pressure, but after using $R^{2}$ and adjusted $R^{2}$ the relationship was no longer significant. According to a report by Zhou et al. [29], an elevated serum insulin level is associated with increased sympathetic nerve activity, increased renal sodium retention, the induction of proliferation of vascular smooth muscle cells and collagen synthesis leading to elevated blood pressure, which is a risk factor of cardiovascular disease. The lack of correlation between systolic blood pressure and the insulin resistance index in this study is likely due to the fact that the YWFH subjects were of normal weight and their insulin resistance index was still within the clinical decision limits.

The relationship of $\operatorname{Lp}(\mathrm{a})$ concentrations with insulin resistance remains controversial and unproven. Some studies reported no association between insulin levels and $L p(a)$ concentration $[30,31]$, while others reported a negative relationship with $\operatorname{Lp}(\mathrm{a})$ concentrations $[32,33]$. Studies have shown that the controversies in the correlation of $\mathrm{Lp}(\mathrm{a})$ against parameters of glycaemia and lipid metabolism is not surprising given that the systemic Lp(a) concentrations are highly genetically determined with little influence from dietary, environmental, and physiological factors [34-36].

This study also showed no correlation between the insulin resistance index and other lipid atherogenic markers such as total cholesterol, HDL-C, TG, LDL-C, ApoB, ApoA-1, and the ApoB/ApoA-1 ratio in YWFH. This contradicts reports from studies by Sierra-Johnson et al. 
[37] and Makaridze et al. [38], where insulin resistance was significantly associated with the ApoB/ApoA-1 ratio and other lipid indices. Makaridze et al. [38] studied nondiabetic populations of Caucasian origin with a mean age of 45 years and reported that insulin resistance was positively correlated with age, ApoB, ApoB/ApoA-1 ratio, LDL-C, fasting insulin, and total cholesterol, and negatively correlated with HDL-C and ApoA-1 in both sexes (all $p<0.001$ ). Sierra-Johnson et al. [37] studied non-diabetic populations in the USA with a mean age of 47 years and concluded that the ApoB/ApoA-1 ratio is significantly associated with insulin resistance in non-diabetic subjects, independently of the traditional risk factors, metabolic syndrome components, and inflammatory risk factors. This suggests that insulin resistance rises with age and its association with these lipid atherogenic markers become significant as the individual grows older. In this study, the study population was young and apparently healthy, made up of non-diabetics with no cardiovascular disease. This may explain the lack of correlation between the insulin resistance index and lipid markers in them.

\section{Conclusion}

This study has demonstrated that a positive family history of type 2 diabetes mellitus is associated with a higher insulin resistance index and elevated atherogenic lipid biomarkers; thus, there is overall increased risk of cardiovascular disease even in normoglycaemic youths with a family history of diabetes. The lack of association between insulin resistance index and lipid biomarkers indicates that the insulin resistance index might not be a reliable indicator for lipid biomarkers in a non-diabetic of a younger age group, as defective insulin action and the associated lipid abnormalities evolve over time.

\section{Acknowledgements}

We acknowledge the resident doctors, nurses, and other staffs of the Metabolic Research Ward, University College Hospital, Ibadan, Nigeria, for their assistance during the OGTT procedure.

\section{Statement of Ethics}

Subjects gave their written informed consent to take part in the study, and recruitment was entirely voluntary at no cost to the participants. All information collected in this study was given code numbers and no names were recorded. The study protocol was approved by the institute's committee on human research. No animals were used in this study.

\section{Disclosure Statement}

The authors have no conflicts of interest to declare.

\section{Funding Sources}

We certify that this article has not been funded by any funding agency.

\section{Author Contributions}

All authors contributed to this study and the preparation of the manuscript.

\section{References}

1 Isara AR, Okundia PO. The burden of hypertension and diabetes mellitus in rural communities in southern Nigeria. Pan Afr Med J. 2015 Feb;20:103.

2 Cimmino G, Ciccarelli G, Alberto M, Michele C, Paolo G. High density lipoprotein cholesterol increasing therapy: The unmet cardiovascular need. Transl Med UniSa. 2015;12(6):29-40.

3 Orringer CE. Non-HDL cholesterol, ApoB and LDL particle concentration in coronary heart disease risk prediction and treatment. Clin Lipidol. 2013;8(1):69-79.

4 Masson W, Siniawski D, Lobo M, Molinero G, Giorgi M, Huerín M. Association between LDL-C, Non HDL-C, and Apolipoprotein B Levels with Coronary Plaque Regression. Arq Bras Cardiol. 2015 Jul;105(1):11-9.
5 Hong LF, Yan XN, Fan Y, Wu Q, Luo SH, Yang $\mathrm{B}$, et al. Is the ratio of apoB/apoA-1 the best predictor for the severity of coronary artery lesions in Chinese diabetics with stable angina pectoris? An assessment based on Gensini scores. J Geriatr Cardiol. 2015 Jul; 12(4):402-9.

6 Willeit P, Kiechl S, Kronenberg F, Witztum JL, Santer P, Mayr M, et al. Discrimination and net reclassification of cardiovascular risk with lipoprotein(a): prospective 15-year outcomes in the Bruneck Study. J Am Coll Cardiol. 2014 Sep;64(9):851-60.

7 Kaur J. A comprehensive review on metabolic syndrome. Cardiol Res Pract. 2014;2014: 943162.
$8 \mathrm{Wu}$ L, Parhofer KG. Diabetic dyslipidemia. Metabolism. 2014 Dec;63(12):1469-79.

9 Manjunath CN, Rawal JR, Irani PM, Madhu K. Atherogenic dyslipidemia. Indian J Endocrinol Metab. 2013 Nov;17(6):969-76.

10 Adeleye JO, Abbiyesuku FM. Glucose and insulin responses in offspring of Nigerian Type 2 diabetics. Afr J Med Med Sci. 2002 Sep; 31(3):253-7.

11 Isganaitis E, Woo M, Ma H, Chen M, Kong W, Lytras A, et al. Developmental programming by maternal insulin resistance: hyperinsulinemia, glucose intolerance, and dysregulated lipid metabolism in male offspring of insulin-resistant mice. Diabetes. 2014 Feb; 63(2):688-700 
12 Fiorentino TV, Succurro E, Arturi F, Giancotti A, Peronace C, Quirino A, et al. Serum IgG2 levels are specifically associated with wholebody insulin-mediated glucose disposal in non-diabetic offspring of type 2 diabetic individuals: a cross-sectional study. Sci Rep. 2018 Sep;8(1):13616.

13 Adeleye JO, Abbiyesuku FM. Anthropometric charasteristics of offspring of Nigerian type 2 diabetics. Niger J Clin Pract. 2002;5(2): $75-80$.

14 Zafar U, Asrar A, Gohar B. Anthropometric parameters of central obesity in non-diabetic offspring of type 2 diabetics and non-diabetic offspring of non-diabetics. Pak J Med Health Sci. 2015;9(3):801-3.

15 Ryder JR, Dengel DR, Jacobs DR Jr, Sinaiko AR, Kelly AS, Steinberger J. Relations among adiposity and insulin resistance with flowmediated dilation, carotid intima-media thickness, and arterial stiffness in children. J Pediatr. 2016 Jan;168:205-11.

16 Frohnert BI, Jacobs DR Jr, Steinberger J, Moran A, Steffen LM, Sinaiko AR. Relation between serum free fatty acids and adiposity, insulin resistance, and cardiovascular risk factors from adolescence to adulthood. Diabetes. 2013 Sep;62(9):3163-9.

17 Fan H, Li X, Zheng L, Chen X, Lan Q, Wu H, et al. Abdominal obesity is strongly associated with Cardiovascular Disease and its Risk Factors in Elderly and very Elderly Communitydwelling Chinese. Sci Rep. 2016 Feb;6(1): 21521.

18 Mohammadifard N, Nazem M, Sarrafzadegan N, Nouri F, Sajjadi F, Maghroun M, et al. Body mass index, waist-circumference and cardiovascular disease risk factors in Iranian adults: isfahan healthy heart program. J Health Popul Nutr. 2013 Sep;31(3):388-97.

19 Chung JO, Cho DH, Chung DJ, Chung MY. Associations among body mass index, insulin resistance, and pancreatic $\beta$-cell function in Korean patients with new-onset type 2 diabetes. Korean J Intern Med (Korean Assoc Intern Med). 2012 Mar;27(1):66-71.

20 Shetty HV, Usha SM, Chandrika N, Manjula KS. Influence of obesity and family history of type 2 diabetes mellitus on serum ferritin and insulin levels in young adults. IBRR. 2014; 2(4):168-77.
21 Rahim M, Qureshi MA, Sharafat S, et al. Lipid profile and growth indicators among offspring's of diabetic parents in Karachi, Pakistan. J Diabetes Metab. 2014;5(10):443-8.

22 Moon JH, Roh E, Oh TJ, Kim KM, Moon JH, Lim S, et al. Increased risk of metabolic disorders in healthy young adults with family history of diabetes: from the Korea National Health and Nutrition Survey. Diabetol Metab Syndr. 2017 Mar;9(1):16.

23 Psyrogiannis A, Habeos I, Kyriazopoulou V. Insulin sensitivity and $\mathrm{Lp}(\alpha)$ concentrations in normoglycemic offspring of type 2 diabetic parents. Lipids Health Dis. 2003 Oct;2(1):8.

24 Virani SS, Brautbar A, Davis BC, Nambi V, Hoogeveen RC, Sharrett AR, et al. Associations between lipoprotein(a) levels and cardiovascular outcomes in black and white subjects: the Atherosclerosis Risk in Communities (ARIC) Study. Circulation. 2012 Jan; 125(2):241-9.

25 Khera AV, Everett BM, Caulfield MP, Hantash FM, Wohlgemuth J, Ridker PM, et al. Lipoprotein(a) concentrations, rosuvastatin therapy, and residual vascular risk: an analysis from the JUPITER Trial (Justification for the Use of Statins in Prevention: an Intervention Trial Evaluating Rosuvastatin). Circulation. 2014 Feb;129(6):635-42.

26 Hashemi M, Saadat M, Behjati M, Kelishadi R. Comparison of serum apolipoprotein levels of diabetic children and healthy children with or without diabetic parents. Cholesterol. 2012;2012:490381.

27 Savas Erdeve S, Simsek E, Dallar Y, Biyikli Z. Utility of ApoB/ApoA1 ratio for the prediction of cardiovascular risk in children with metabolic syndrome. Indian J Pediatr. 2010 Nov;77(11):1261-5.

28 Kaneva AM, Potolitsyna NN, Bojko ER, Odland JO. The apolipoprotein B/apolipoprotein A-I ratio as a potential marker of plasma atherogenicity. Dis Markers. 2015;2015: 591454.
29 Zhou MS, Wang A, Yu H. Link between insulin resistance and hypertension: what is the evidence from evolutionary biology? Diabetol Metab Syndr. 2014 Jan;6(1):12.

30 Månsson M, Kalies I, Bergström G, Schmidt C, Legnehed A, Hultén LM, et al. Lp(a) is not associated with diabetes but affects fibrinolysis and clot structure ex vivo. Sci Rep. 2014 Jun;4(1):5318.

31 Todoric J, Handisurya A, Leitner K, Harreiter J, Hoermann G, Kautzky-Willer A. Lipoprotein(a) is not related to markers of insulin resistance in pregnancy. Cardiovasc Diabetol. 2013 Oct;12(1):138.

32 Mora S, Kamstrup PR, Rifai N, Nordestgaard BG, Buring JE, Ridker PM. Lipoprotein(a) and risk of type 2 diabetes. Clin Chem. 2010 Aug;56(8):1252-60.

33 Ding L, Song A, Dai M, Xu M, Sun W, Xu B, et al. Serum lipoprotein (a) concentrations are inversely associated with T2D, prediabetes, and insulin resistance in a middle-aged and elderly Chinese population. J Lipid Res. 2015 Apr;56(4):920-6.

34 Fontana P, Mooser V, Bovet P, Shamlaye C, Burnand B, Lenain V, et al. Dose-dependent inverse relationship between alcohol consumption and serum Lp(a) levels in black African males. Arterioscler Thromb Vasc Biol. 1999 Apr;19(4):1075-82.

35 Tsimikas S. A Test in Context: Lipoprotein(a): Diagnosis, Prognosis, Controversies, and Emerging Therapies. J Am Coll Cardiol. 2017 Feb;69(6):692-711.

36 Kronenberg F. Human Genetics and the Causal Role of Lipoprotein(a) for Various Diseases. Cardiovasc Drugs Ther. 2016 Feb; 30(1):87-100.

37 Sierra-Johnson J, Romero-Corral A, Somers VK, Lopez-Jimenez F, Walldius G, Hamsten A, et al. ApoB/apoA-I ratio: an independent predictor of insulin resistance in US non-diabetic subjects. Eur Heart J. 2007 Nov;28(21): 2637-43

38 Makaridze Z, Giorgadze E, Asatiani K. Association of the apolipoprotein b/apolipoprotein a-I ratio, metabolic syndrome components, total cholesterol, and low-density lipoprotein cholesterol with insulin resistance in the population of georgia. Int J Endocrinol. 2014;2014:925650. 